\title{
Clinical and Epidemiological Characteristics of COVID-19 in the Barros Luco Hospital Emergency Unit: the First 164 Patients
}

\author{
Giancarlo Zuccone, ${ }^{1 *}$ Valentina Albornoz, ${ }^{1}$ Helga Ibáñez, ${ }^{1}$ Raúl Betancur, ${ }^{2}$ Julio Matute ${ }^{3}$ \\ ${ }^{1}$ Inmates on elective emergency rotation in the HBLT Emergency Unit, Diego Portales University, Chile \\ ${ }^{2}$ Emergency Physician, Technical Chief of the HBLT Emergency Unit, Chile \\ ${ }^{3}$ Emergency and Intensive Care Physician, Head of the HBLT Emergency Unit, Chile
}

\begin{abstract}
Our country was one of many hit by the new coronavirus pandemic, of which its clinical manifestation, mortality rate, laboratory and imaging findings are not still fully understood and in research. This document pretends to elaborate a presentation profile-which includes clinical manifestation, radiologic and laboratory findings-of 164 SARS-CoV2 (+) patients that concurred to the Emergency Service in Barros Luco-Trudeau Hospital. This retrospective-descriptive research paper shows that the most common SARS-CoV2 (+) patient consulting on this ER was predominantly young, not necessarily having a known contact for SARS-CoV2 nor risk factors for complications related to COVID-19. Clinically, dry coughing, cephalalgy and myalgia were more often referred. $74 \%$ of patients required house-care only, without any further complications during the disease. Nonetheless, $6 \%$ of patients regarding this sample required treatment in the ICU in some point after from onset of the symptoms. In the group of the ICU patients, the most remarkable laboratory differences when compared to the house-care group were show on D-dimer and ferritin blood levels.
\end{abstract}

Keywords: COVID-19, SARS-COV2, Coronavirus, Pandemic

\section{Introduction}

Since December 2019, there has been a record of the first patients infected with a new type of enveloped beta coronavirus RNA in Wuhan, China, called Severe Acute Respiratory Syndrome Corovairus2 (SARS-CoV2).$^{1-3}$ Its clinical presentation would vary, from the asymptomatic carrier to the critically ill patient with interstitial pneumonia and severe ARDS. ${ }^{1,45}$ It would be transmitted by droplets from a carrier patient (when talking, coughing or sneezing) to a non-carrier. ${ }^{1,6}$ The virus would be viable for a variable time on different surfaces, being able to adhere to the skin of the previously non-carrier patient, which would explain the infections without apparent SARS-CoV2(+) contacts. ${ }^{2,3}$ Recent evidence postulates that the virus has an incubation period of up to 14 days, from its inoculation to the presentation of symptoms, with an average of 4 to 5 days, and its risk of transmission would depend on several factors, including the time of exposure to a symptomatic patient and/or carrier, viral load, personal protection measures, use of a mask in the symptomatic or suspected patient, maintenance of the defined social distance between 1 and 1.5 meters between people, overcrowding and/or recent participation in crowds and in-hospital exposure. ${ }^{1,3,7}$ Our environment is no stranger to the pandemic, having confirmed cases since the beginning of March. However, there is little information published and available in our environment about the clinical presentation of patients who consult and their comorbidities, alterations in laboratory tests and other data, such as characteristics of patients hospitalized in the ward or in the PCU.

Therefore, in the Barros Luco Hospital Emergency Unit and in conjunction with various specialties, different medical guidelines have been developed, highlighting the presentation of different
Quick Response Code:

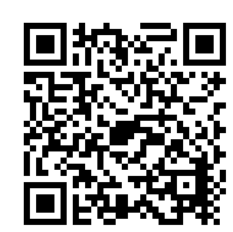

*Corresponding author: Giancarlo Zuccone Z, Inmates on elective emergency rotation in the HBLT Emergency Unit, Diego Portales University, Chile

Received: 25 May, 2021

Published: 08 June, 2021

Citation: Giancarlo Z, Valentina A, Helga I, Raúl B, Julio M. Clinical and Epidemiological Characteristics of COVID-19 in the Barros Luco Hospital Emergency Unit: the First 164 Patients. Curr Inv CIn Med Res. 2021;1(2):1-6. DOI: 10.53902/CICMR.2021.01.000506 
clinical cases according to their initial epidemiological and clinical antecedents; this in order to find the profile of the patient who can consult in a highly complex emergency room with SARS-CoV2 disease.We present this document with the intention of providing data given the situation, as observed in our Emergency Unit, looking for common characteristics among SARS-CoV2 (+) patients that allow establishing the most prevalent clinical presentations of the disease.

\section{Materials and Methods}

This study is intended to be retrospective and descriptive, seeking to describe the epidemiological and clinical characteristics of the first SARS-CoV2(+) patients.The present review was carried out in the Emergency Unit of Hospital Barros Luco between March 12 and April 27, 2020, achieving n of 164 patients. All the Urgent Care Data (DAU) of the patients who were treated in the COVID Box of the Emergency Unit with PCR(+) for the virus were reviewed. Laboratory tests and images that would have been taken by internal protocol according to current medical guidelines were also reviewed in the care of suspected COVID patients. Said information was stored and arranged in an Excel® sheet to later be analyzed using percentages and measures of central tendency.In parallel, the cases that were managed on an outpatient basis were followed up through a Health Team led by a doctor, via telephone calls and applying a survey to define the future controls of SARS-CoV2(+) in outpatient management. In hospitalized patients, clinical, laboratory and imaging parameters were also monitored in order to have a better perspective of the evolution of the cases without intervening in the management and medical study regarding their complexity. This work is approved by the Healthcare Ethics Committee of the Barros Luco Hospital according to the current Quality Protocol. ${ }^{8-10}$

\section{Results}

The sample consists of 164 patients with CRP (+) for SARSCoV2, of which 2 (1.22\% of the sample) turned out to be asymptomatic. All the rest (162 patients, 98.78\%) presented varied symptoms and signs, to be characterized later.

\section{Demographic characteristics}

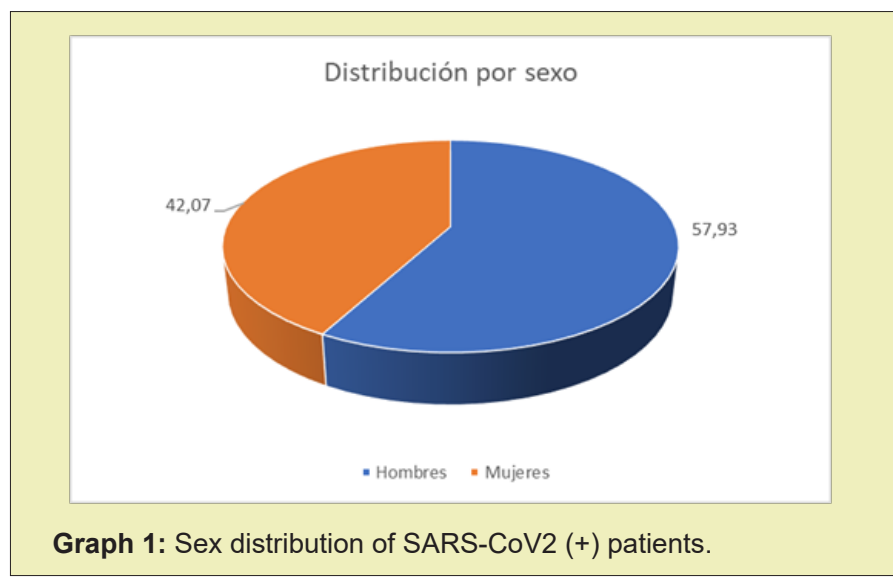

Of this sample, 95 patients (57.93\%) were male, and 69 (42.07\%) were female (Graph 1). The youngest patient consulted was 16 years old, while the oldest was 92 . The mean age of these
SARS-CoV2 (+) patients was 46 years, with a median of 45 years and a mode of 32. The distribution According to the age ranges of these patients, it is detailed in Table 1 and Graph 2. The daily and accumulated infections of SARS CoV2 $(+)$ patients in the data collection interval are also presented in Table 2 and Graph 3.

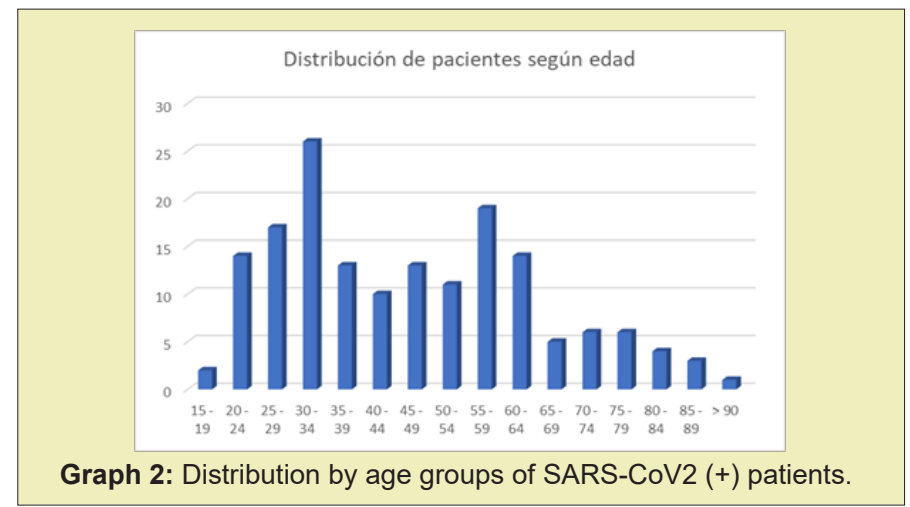

Table 1: Age distribution of SARS- CoV2 (+) patients, with mean, median and mode.

\begin{tabular}{|c|c|c|}
\hline Ranges & Frequency & Percentage \\
\hline $15-19$ & 2 & 1.22 \\
\hline $20-24$ & 14 & 8.54 \\
\hline $25-29$ & 17 & 10.37 \\
\hline $30-34$ & 26 & 15.85 \\
\hline $35-39$ & 13 & 7.93 \\
\hline $40-44$ & 10 & 6.10 \\
\hline $45-49$ & 13 & 7.93 \\
\hline $50-54$ & 11 & 6.71 \\
\hline $55-59$ & 19 & 11.59 \\
\hline $60-64$ & 14 & 8.54 \\
\hline $65-69$ & 5 & 3.05 \\
\hline $70-74$ & 6 & 3.66 \\
\hline $75-79$ & 6 & 3.66 \\
\hline $80-84$ & 4 & 2.44 \\
\hline $85-89$ & 3 & 1.83 \\
\hline$>90$ & 1 & 0.61 \\
\hline Average & Median & Mode \\
\hline 46 & 45 & 32 \\
\hline
\end{tabular}

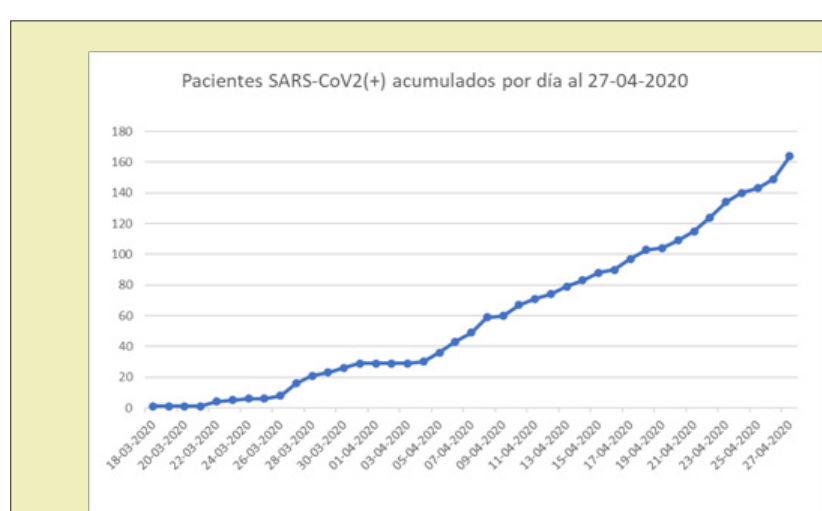

Graph 3: Curve of SARS-CoV $2(+)$ patients accumulated per day until 04-27-2020. 
Table 2: Percentage distribution of SARS- CoV2 (+) patients by commune of residence.

\begin{tabular}{|c|c|}
\hline Commune & Percentage \\
\hline San Miguel & 16.15 \\
\hline Lo Espejo & 13.04 \\
\hline Pedro Aguirre Cerda & 11.8 \\
\hline San Joaquín & 11.8 \\
\hline La Cisterna & 8.7 \\
\hline Santiago & 8.7 \\
\hline San Bernardo & 6.83 \\
\hline El Bosque & 4.97 \\
\hline Not Registered & 3.73 \\
\hline Maipú & 1.86 \\
\hline Pudahuel & 1.86 \\
\hline Estación Central & 1.24 \\
\hline Quilicura & 1.24 \\
\hline Angol & 0.62 \\
\hline Buin & 0.62 \\
\hline Huechuraba & 0.62 \\
\hline Iquique & 0.62 \\
\hline Florida & 0.62 \\
\hline La Pintana & 0.62 \\
\hline Padre Hurtado & 0.62 \\
\hline Padre Las Casas & 0.62 \\
\hline Providencia & 0.62 \\
\hline Quinta normal & 0.62 \\
\hline Renca & 0.62 \\
\hline San Ramon & 0.62 \\
\hline Talcahuano & 0.62 \\
\hline
\end{tabular}

\section{Clinical characteristics}

The clinical presentation of SARS CoV2 patients is varied, with combinations of respiratory and non-respiratory symptoms. The most frequent symptom was dry cough, reported in $46.95 \%$ of cases (77 patients), followed by headache in $42.07 \%$ of cases (69 patients) and myalgia in $41.46 \%$ (68 patients). The details of the referred symptoms and their frequency of presentation are shown in Graph 4 and Table 3. On the other hand, 62 patients (37.8\%) reported in their anamnesis having some comorbidity or risk factor that lead to complications associated with SARS-CoV2 infection and that could require eventual hospital management, such as: HT and others cardiovascular risk factors, previous pulmonary disease and/or chronic active or passive smoking, diabetes, CKD, obesity and immunosuppression conditions. The other 102 patients $(62.2 \%)$ either did not report underlying pathologies or reported some comorbidity that did not correspond to the group of pathologies mentioned. The mean time from the onset of symptoms to the ED consultation was 4 days, with a minimum time of 1 day from the onset of symptoms and a maximum of 21 days of symptoms prior to the consultation. ${ }^{11-15}$
Table 3: Count of SARS- CoV2 (+) patients per day and accumulated as of 04-27-2020.

\begin{tabular}{|c|c|c|}
\hline Date & Pcs. COVID (+) accumulated & Pcs. COVID (+) / day \\
\hline 03-18-2020 & 1 & 1 \\
\hline 03-19-2020 & 1 & 0 \\
\hline $20-03-2020$ & 1 & 0 \\
\hline 03-21-2020 & 1 & 0 \\
\hline $03-22-2020$ & 4 & 3 \\
\hline 03-23-2020 & 5 & 1 \\
\hline 03-24-2020 & 6 & 1 \\
\hline $03-25-2020$ & 6 & 0 \\
\hline 03-26-2020 & 8 & 2 \\
\hline 03-27-2020 & 16 & 8 \\
\hline 03-28-2020 & 21 & 5 \\
\hline 03-29-2020 & 2.3 & 2 \\
\hline 03-30-2020 & 26 & 3 \\
\hline $03 / 31 / 2020$ & 29 & 3 \\
\hline 01-04-2020 & 29 & 0 \\
\hline 02-04-2020 & 29 & 0 \\
\hline 03-04-2020 & 29 & 0 \\
\hline 04-04-2020 & 30 & 1 \\
\hline 05-04-2020 & 36 & 6 \\
\hline 06-04-2020 & 43 & 7 \\
\hline 07-04-2020 & 49 & 6 \\
\hline 08-04-2020 & 59 & 10 \\
\hline 09-04-2020 & 60 & 1 \\
\hline 04-10-2020 & 67 & 7 \\
\hline 04-11-2020 & 71 & 4 \\
\hline $12-04-2020$ & 74 & 3 \\
\hline 04-13-2020 & 79 & 5 \\
\hline 04-14-2020 & 83 & 4 \\
\hline 04-15-2020 & 88 & 5 \\
\hline 04-16-2020 & 90 & 2 \\
\hline 04-17-2020 & 97 & 7 \\
\hline 04-18-2020 & 103 & 6 \\
\hline 04-19-2020 & 104 & 1 \\
\hline $20-04-2020$ & 109 & 5 \\
\hline 21-04-2020 & 115 & 6 \\
\hline $04-22-2020$ & 124 & 9 \\
\hline 04-23-2020 & 134 & 10 \\
\hline 04-24-2020 & 140 & 6 \\
\hline $04-25-2020$ & 143 & 3 \\
\hline 04-26-2020 & 149 & 6 \\
\hline $27-04-2020$ & 164 & 2 \\
\hline
\end{tabular}




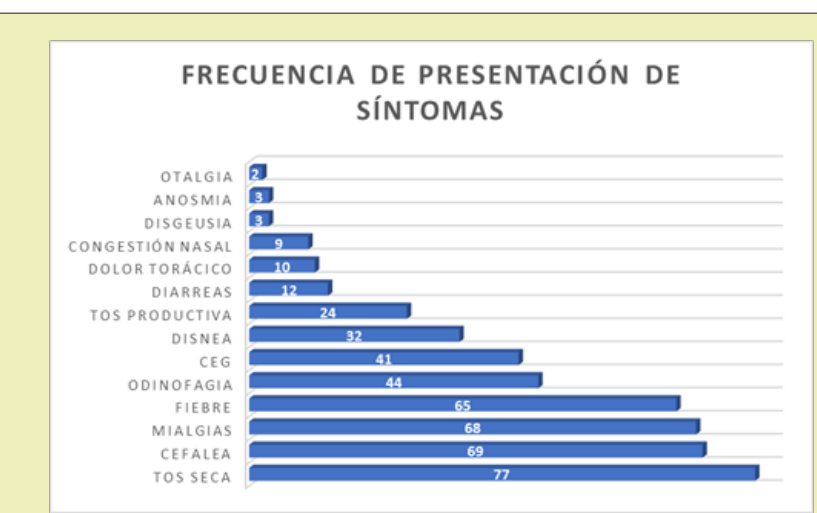

Graph 4: Frequency of presentation of symptoms referred by SARS-CoV2 (+) patients at the time of consultation in the Emergency Unit.

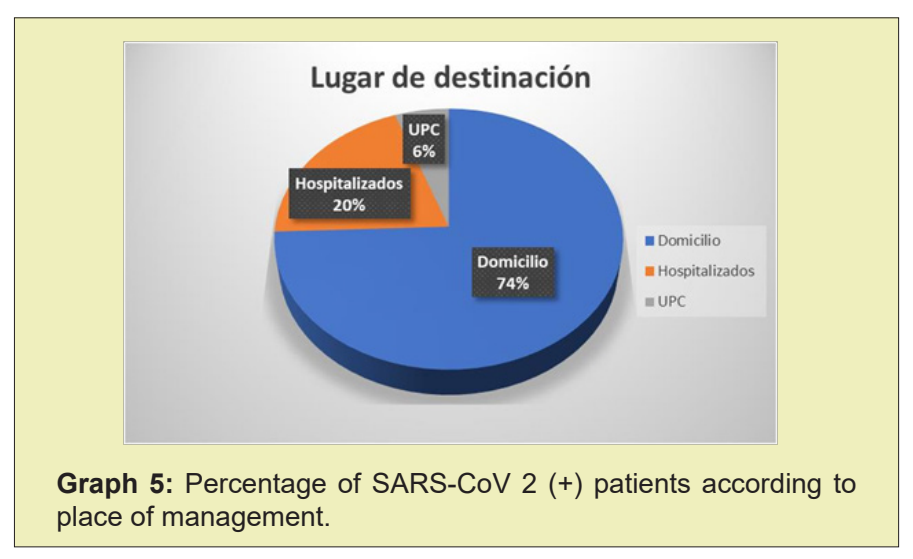

Of this sample, 75 patients $(45.73 \%)$ reported having contact with a SARS-CoV2 (+) patient prior to the consultation and/ or some trip outside the MR but within the country. None referred trips abroad. The other 89 patients (54.27\%) denied contacts with SARS-CoV2 positive patients and/or travel. Of this sample, 122 patients $(74.39 \%)$ were under exclusive home management or were discharged without requiring subsequent hospital management. 33 patients (20.12\%) required hospital ward management at some point in the evolution of the SARS-CoV2 disease, and a total of 9 patients (5.49\%) required management in the UPC(Unidad de Paciente Crítico) (Graph 5) (Table 4).

Table 4: Frequency of presentation and percentage of symptoms reported by SARS- CoV2 $(+)$ patients at the time of consultation in the Emergency Unit.

\begin{tabular}{|c|c|c|}
\hline Symptoms & Presentation frequency & Percentage \\
\hline Dry cough & 77 & 46.95 \\
\hline Headache & 69 & 42.07 \\
\hline Myalgia & 68 & 41.46 \\
\hline Fever & 65 & 39.63 \\
\hline Odynophagia & 44 & 26.83 \\
\hline CEG & 41 & 25.00 \\
\hline Dyspnoea & 32 & 19.51 \\
\hline Productive cough & 24 & 14.63 \\
\hline Diarrhea & 12 & 7.32 \\
\hline Chest pain & 10 & 6.10 \\
\hline Nasal congestion & 9 & 5.49 \\
\hline Dysgeusia & 3 & 1.83 \\
\hline Anosmia & 3 & 1.83 \\
\hline Ear pain & 2 & 1.22 \\
\hline
\end{tabular}

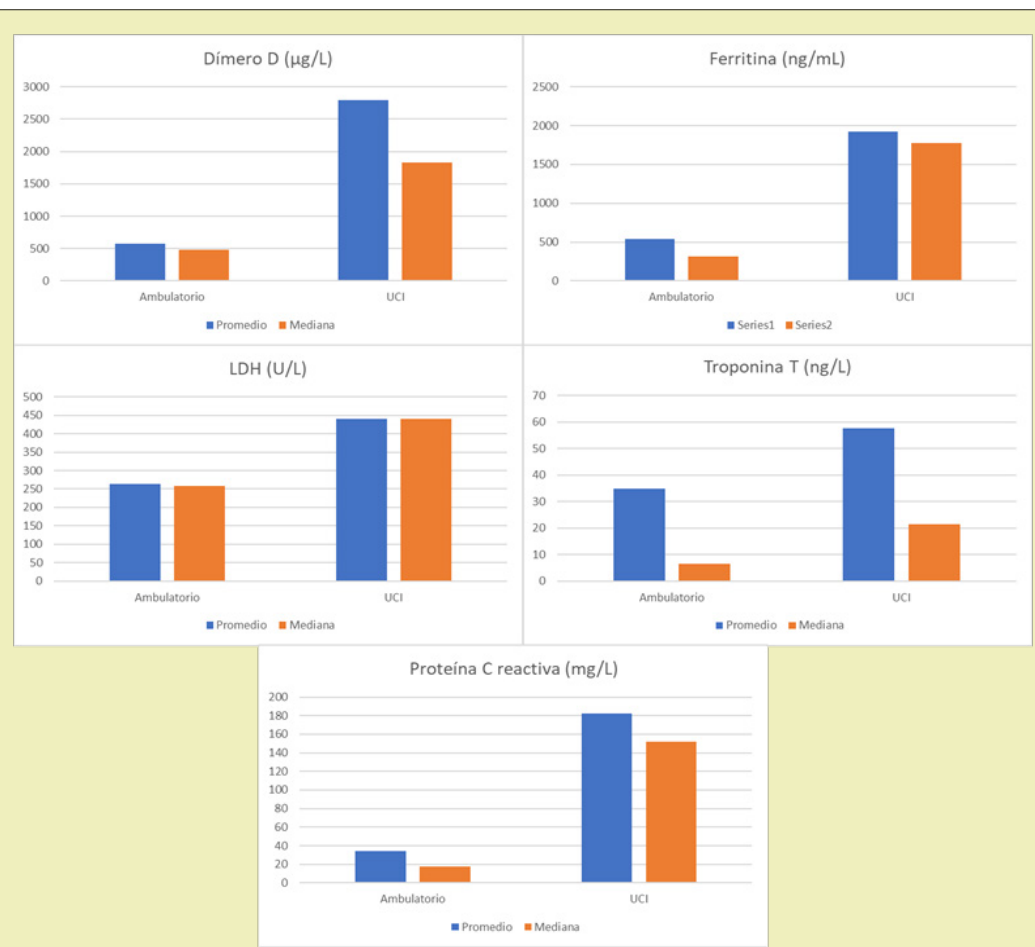

Graph 6: Comparison of different laboratory parameters in outpatients (left columns) and in ICU patients (right columns). In blue are the average values and in orange the median values. 


\section{Imaging characteristics}

Of the total number of patients, imaging tests (chest CT) were taken in 48 cases (29.3\% of the total). Of these, 39 presented unilateral or bilateral diffuse ground glass pattern, consistent with SARSCoV2 infection (equivalent to $81.2 \%$ of the imaging tests taken), and 9 that did not present such alterations (18.8\%). An example of the alterations described in 2 of these patients is seen in Figure 1. Of the total imaging exams taken, $38(71.2 \%$ of the total imaging exams) correspond to patients who required hospital and/or ICU management. Of these, 35 showed alterations concordant with SARS-CoV2 infection (92.1\%), 3 had images without alterations attributable to SARS-CoV2 (7.9\%)(Graph 6).

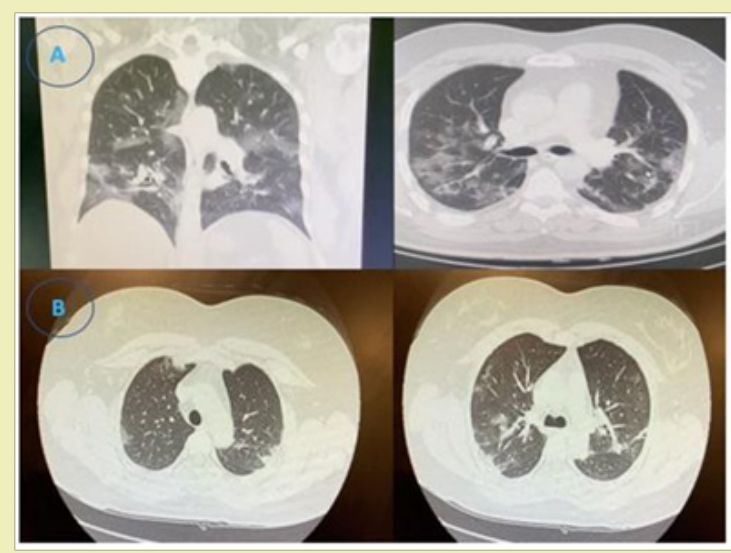

Figure 1: Imaging characteristics of COVID-19: ground glass opacities, consolidations and pattern in paving stone or crazy paving. A) Initial images of a patient who is hospitalized in the UPC with need for IMV 36 hours after admission. B) Initial images of a patient with outpatient management and follow-up with good clinical evolution.

\section{Features lab}

Regarding the laboratory tests, we compared the values of C-reactive protein (CRP) and ferritin as inflammatory markers, troponin $\mathrm{T}$ as a marker of myocardial damage, LDH in correlation with lung damage and D-dimer (DD) as a marker of bleeding disorder, using mean and median as measures of central tendency. Their comparison both in average and median are represented in Figure 7.D- dimerthe maximum value for DD recorded in patients in outpatient management was $1811 \mu \mathrm{g} / \mathrm{L}$, with an average of $574 \mu \mathrm{g} / \mathrm{L}$ and a median of $484 \mu \mathrm{g} / \mathrm{L}$. This versus patients managed in the ICU, with a maximum recorded value of $6558 \mu \mathrm{g} / \mathrm{L}$, an average of $2791 \mu \mathrm{g} / \mathrm{L}$, and a median of $1826 \mu \mathrm{g} / \mathrm{L}$.

\section{Ferritin}

The maximum value for ferritin recorded in patients in outpatient management was $1935 \mathrm{ng} / \mathrm{mL}$, with an average of 540ng/ $\mathrm{mL}$ and a median of $315 \mathrm{ng} / \mathrm{mL}$. In patients managed in the ICU, the maximum value recorded was $5190 \mathrm{ng} / \mathrm{mL}$, with an average of $1925 \mathrm{ng} / \mathrm{mL}$ and a median of $1776 \mathrm{ng} / \mathrm{mL}$.

\section{LDH}

The maximum value for LDH in outpatients was 421U/L, with a mean of $264 \mathrm{U} / \mathrm{L}$ and a mean of $259 \mathrm{U} / \mathrm{L}$. In patients managed in the ICU, the maximum recorded was $648 \mathrm{U} / \mathrm{L}$, with an average of $440 \mathrm{U} / \mathrm{L}$ and a median of $441 \mathrm{U} / \mathrm{L}$.

\section{Troponin T}

The maximum value for troponin $\mathrm{T}$ in patients in outpatient management was $283 \mathrm{ng} / \mathrm{L}$, with a mean of $35 \mathrm{ng} / \mathrm{L}$ and a median of $7 \mathrm{ng} / \mathrm{L}$. In patients managed in the ICU, the maximum was $234 \mathrm{ng} / \mathrm{L}$, an average of 58ng/L, and a median of $22 \mathrm{ng} / \mathrm{L}$ and PCRThe maximum CRP value in outpatients was $100.6 \mathrm{mg} / \mathrm{L}$, with a mean of $34 \mathrm{mg} / \mathrm{L}$ and a mean of $18 \mathrm{mg} / \mathrm{L}$. Versus patients managed in the ICU, with a maximum value of $550 \mathrm{mg} / \mathrm{L}$, an average of $182 \mathrm{mg} / \mathrm{L}$ and a median of $152 \mathrm{mg} / \mathrm{L}^{16-19}$

\section{Discussion}

This work is intended to be merely descriptive and therefore its results may not necessarily be extrapolated to the reality of other highly complex Emergency Units at the national level. We currently know that there is a non-negligible percentage of asymptomatic carriers with the capacity to infect. ${ }^{1,3,4}$ Most of the patients who consulted with symptoms of SARS-CoV2 were men. The age distribution of the patients who consulted was quite wide, with its average around 45 years and a peak in the group between 30 and 34 years, and there is a tendency not to report a known positive contact for SARS-CoV2 prior to PCR diagnosis. All of the above could have to do with the work contexts of each patient, in which perhaps measures such as tele-working did not exist or it was not possible to implement, in association with other unfavorable conditions, such as forced use of public transport, need for work and/or supply in places with a high concentration of people such as free fairs, and housing conditions that do not allow effective isolation among its inhabitants, such as shared rooms and limited availability of exclusive bathrooms for infected people. ${ }^{2,4,7}$ The loss of traceability of the contagion chain could be explained both by asymptomatic carriers with contagion capacity in association with the crowding factors of people already described. ${ }^{6,7}$ The contagion curve in this initial stage of the pandemic shows, in its first phase, an apparently linear characteristic, with a break in its slope and on the rise around April 5. The average time of presence of symptoms was almost 4 days before consulting, compatible with other similar reports. ${ }^{2,3,7}$

Regarding imaging and laboratory examinations, there seems to be a certain correlation between the severity of the patient (defined by their level of management, whether it was at home or in a hospital setting and, in the latter, in the ward versus ICU), the imaging alterations and the imaging values and alterations present. The presence of high ferritin and CRP, both inflammatory markers, grew as more specialized medical management was required. The same occurs with the presence of alterations in other parameters, especially D-Dimer and troponin $\mathrm{T}$, which could translate into greater coagulation and myocardial compromise in the most seriously ill patients. Elevation of these parameters were more common and marked as the level of management progressed (from home to ICU), and the available evidence cites them as markers of severity and poor prognosis, consistent with what was seen in this sample., ${ }^{3,48}$ Among the limitations of this document are the fact that the data obtained were all from compilations subsequent to the moment of the consultation, by reading the DAU and recording the system of examinations requested and if the doctor who performed the care did not record the results. Findings in the DAU, these were not in- 
cluded in the study. Likewise, although there was always an attempt to corroborate and confirm other data at the time of notification, especially the domicile of infected patients, we did find non-concordant data that could affect the quality of this registry.

The $\mathrm{n}$ of patients in the present observational study is rather low given the initial context of the pandemic in the country, which could call into question the conclusions obtained. Comparison with other studies of this nature, with a greater $\mathrm{n}$ of patients and statistical analysis, is necessary for the construction of solid clinical evidence.Among the advantages, it is said that the data collection methodology is highly replicable in other centers, with well-defined objective and subjective parameters, which gives rise to other similar works that facilitate the global statistical analysis of the pandemic to level of the different hospital centers nationwide. The possibility of accessing both the AED, laboratory and imaging tests retrospectively, together with the rapid confirmation of the diagnosis of SARS-CoV2 (+) patients in the initial stages of the pandemic, allowed the development of this work was done quickly and smoothly despite the state of health emergency.

\section{Conclusion}

As a final result, we have that the patient with COVID-19 who consulted in our Emergency Unit was characterized by being mostly young, male, in which non-productive cough, headache and myalgia stood out as the most frequent presenting symptoms, and It was more prevalent that SARS CoV2 (+) patients did not have accurate knowledge of a positive contact with another virus carrier. A good part of this sample required management only at home, although a non-negligible percentage had complications serious enough to require hospitalization in the ICU. Blood parameters such as ferritin, CRP, D-Dimer and Troponin $\mathrm{T}$ are those that seem to be altered to a greater extent in patients with more severe COVID-19 symptoms, so we believe they could be important prognostic markers in patients with SARS disease- Symptomatic CoV2. Likewise, we believe it is necessary to do strict monitoring of those patients whose clinic does not warrant hospitalization at the time of consultation in the emergency service, but who have any of the exposed risk factors and/or alterations in laboratory tests such as those described.

\section{Acknowledgments}

None.

\section{Funding}

This work had no funding of any kind.

\section{Conflicts of Interest}

Author declares that there is no conflict of interest.

\section{References}

1. Wei jie Guan, Zheng yi $\mathrm{Ni}, \mathrm{Yu} \mathrm{Hu}$, et al. Clinical characteristics of coronavirus disease 2019 in China. $N$ Engl J Med. 2020;382:1708-1720.
2. Xiao Wei Xu, Xiao Xin $\mathrm{Wu}$, Xian Gao Jiang, et al. Clinical findings in a group of patients infected with the 2019 novel coronavirus (SARS-Cov-2) outside of Wuhan, China:retrospective case series. BMJ. 2020;368:606.

3. Nanshan Chen, Min Zhou, Xuan Dong, et al. Epidemiological and clinical characteristics of 99 cases of 2019 novel coronavirus pneumonia in Wuhan, China:a descriptive study. Lancet. 2020;395(10223):507-513.

4. Su Shuo, Wong Gary, Shi Wenifeng, et al. Epidemiology, genetic recombination, and pathogenesis of coronaviruses. Trends Microbiol. 2016;24:490-502.

5. Dawei Wang, Bo Hu, Chang Hu, et al. Clinical Characteristics of 138 Hospitalized Patients With 2019 Novel Coronavirus-Infected Pneumonia in Wuhan, China. JAMA. 2020;323(11):1061-1069.

6. Na Zhu, Dingyu Zhang, Wenling Wang, et al. A NovelCoronavirus from Patients with Pneumonia in China, 2019. NEnglJMed.2020;382:727-733.

7. Jun She, Jinjun Jiang, Ling Ye, et al. 2019 novel coronavirus of pneumonia in Wuhan, China:emerging attack and management strategies. Clin Trans Med. 2020;9:19.

8. Weiss Susan, Leibowitz Julian. Coronavirus pathogenesis. Adv Virus Res. 2011;81:85-164.

9. Huang Chaolin, Yeming Wang, Xingwang Li, et al. Clinical features of patients infected with 2019 novel coronavirus in Wuhan, China. Lancet. 2020;395(10223):497-506.

10. Yi Zheng, Chang Xiong, Yuquan Liu, et al. Epidemiological and clinical characteristics analysis of COVID-19 in surrounding areas of Wuhan, Hubei Province in 2020. Pharmacol Res. 2020;157:104821.

11. Huipeng Ge, Xiufen Wang, Xiangning Yuan, et al. The epidemiology and clinical information about COVID-19. Eur J Clin Microbiol Infect Dis. 2020;39(6):1011-1019.

12. Shirley Masse, Lisandru Capai, Natacha Villechenaud, et al. Epidemiology and clinical symptoms related to seasonal coronavirus identified in patients with acute respiratory infections consulting in primary care over six influenza seasons (2014-2020) in France. Viruses. 2020;12(6):630.

13. Jin Jin Zhang, Xiang Dong, Yi Yuan Cao, et al. Clinical characteristics of 140 patients infected with SARS-CoV-2 in Wuhan, China. Allergy. 2020;75 (7):1730-1741.

14. Xiaobo Yang, Yuan Yu, Jiqian $\mathrm{Xu}$, et al. Clinical course and outcomes of critically ill patients with SARS-CoV-2 pneumonia in Wuhan, China:a single-centered, retrospective, observational study. Lancet. 2020;8 (5):475-481.

15. Camila Rothe, Mirjam Schunk, Peter Sothmann, et al. Transmission of 2019-nCoV infection from an asymptomatic contact in Germany. NEJM. 2020;382;10

16. Graham Carlos, Charles Dela Cruz, Bin Cao, et al. Novel Wuhan (2019nCoV) Coronavirus. Am J Respir Crit Care Med. 2020;201 (4):7-8.

17. Tanu Singhal. A review of coronavirus disease-2019 (COVIS-19). Indian J Pediatr. 2020;87(4):281-286.

18. Michelle Holshue, Chas DeBolt, Scott Lindquist, et al. First case of 2019 novel coronavirus in the United States. NEJM. 2020;382:10.

19. Hao Wang, Ran Wei, Guihua Rao, et al. Characteristic CT findings distinguishing 2019 novel coronavirus disease (COVID-19) from influenza pneumonia. Eur Radiol. 2020;Apr 22:1-8. 